\title{
Diskussion zur „Opioid-Krise“ in den USA
}

Aktuelle Berichte über Straf- und Vergleichszahlungen von Opioid-Herstellern im Zusammenhang mit der dramatischen Opioid-Krise in den USA haben auch die Diskussion wieder neu angefacht, was diese Entwicklungen für Europa bedeuten. Expert*innen plädieren für einen rationalen Einsatz von Opioiden in der Schmerzmedizin.

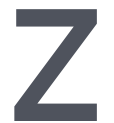

uletzt war es Johnson\&Johnson, hierzulande vertreibt die Firma als Janssen Schmerzmedikamente, die im Zusammenhang mit der sogenannten Opioid-Krise in die Schlagzeilen kam: 230 Millionen Dollar, so ein Vergleich mit dem Bundesstaat New York, muss der Konzern zahlen und in den USA - aber nicht anderswo auf der Welt - aus dem OpioidGeschäft aussteigen. Auch andere Hersteller sahen oder sehen sich in den USA mit hohen Entschädigungsforderungen konfrontiert. Allein Purdue Pharma hatte im Rahmen eines Vergleichs 8,3 Milliarden Dollar bezahlt, um alle Verfahren auf Bundesebene beizulegen. Auch McKinsey als Berater von Purdue bzw. deren Eigentümerfamilie Sackler hat bereits vor einiger Zeit in einem Vergleich einer Zahlung von mehr als 500 Millionen Dollar zugestimmt, um nur einige Beispiele zu nennen.

Die veröffentlichten Daten des Center for Disease Control and Prevention (CDC), einer Einrichtung des US-Gesundheitsministeriums, sind alarmierend: Ein großer Teil der etwa 90.000 Drogentoten, die in den USA 2020 verzeichnet wurden, gehen auf das Konto Opioid-haltiger Analgetika oder illegaler Opioide. Tatsächlich gibt es keinen Zweifel an der Tatsache, dass die Zahl der Opioid-Toten in den USA steigt und die Menge der verschriebenen OpioidAnalgetika nach starken Zuwächsen über mehr als 15 Jahre erst in den vergangenen zwei Jahren eine Abschwächung findet. In Australien sehen die epidemiologischen Daten ähnlich aus.

In der öffentlichen Debatte darüber wird häufig der Missbrauch von Opioiden und der Einsatz dieser Medikamente in der Schmerzmedizin nicht differenziert betrachtet, was auch zu Missverständnissen und Verunsicherungen führen kann. Es ist auch klar zu unterscheiden, ob Opioide medizinisch gegen Schmerzen oder zur Behandlung einer Suchterkrankung eingesetzt werden.

In der Diskussion muss unbedingt berücksichtigt werden, dass es zwischen Nord-

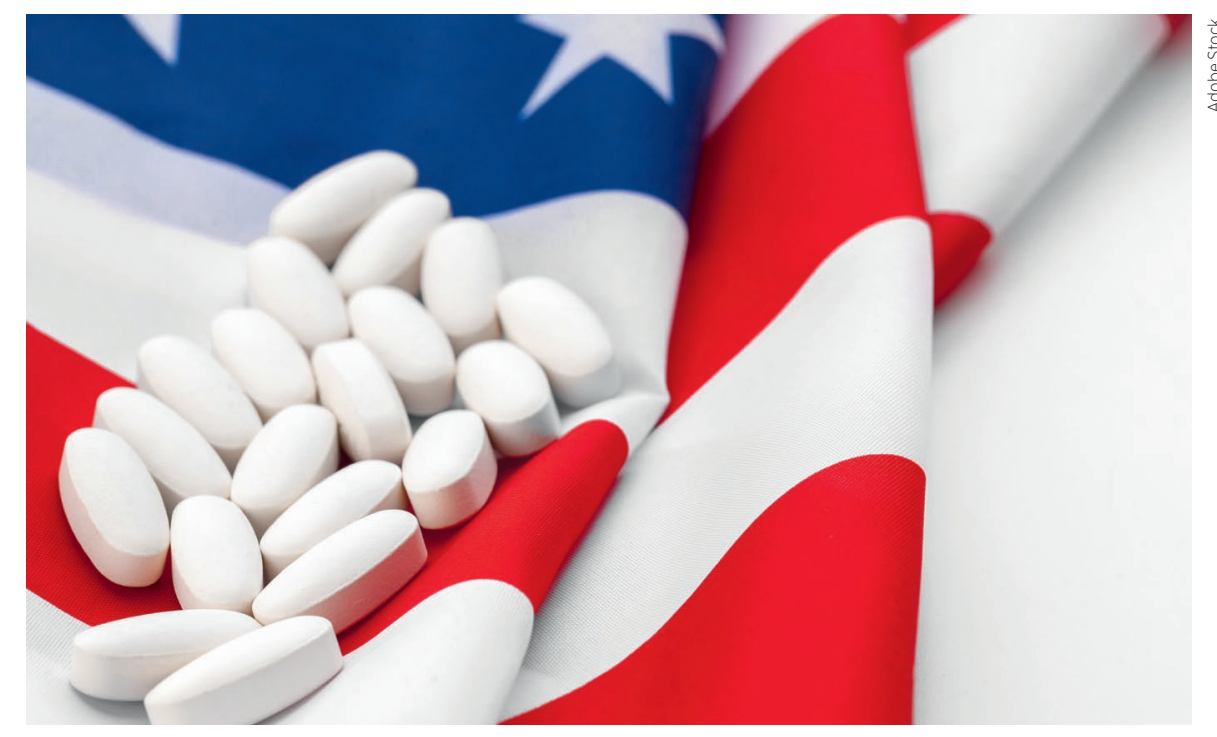

amerika und Europa erhebliche Unterschiede gibt, was den Einsatz von Opioiden betrifft. Anders als in den USA orientiert sich die überwiegende Mehrheit von Ärzt*innen in Europa - und speziell in Österreich strikt an den wissenschaftlichen Empfehlungen. In den USA hingegen werden Opioide leider auch häufig in Situationen verschrieben, in denen nicht zu dieser Substanzgruppe gegriffen werden sollte. So gibt es etwa Untersuchungen, dass in Notfallambulanzen in Washington DC 40 Prozent der Patient*innen, die Schmerzen angeben, beim Erstkontakt ein starkes Opioid erhalten. Ein solcher Umgang trägt zu steigenden Abhängigkeitszahlen bei. Eine Arbeit aus Deutschland zeigt andererseits, dass sich die Opioid-Abhängigkeit in Deutschland - die Situation ist mit Österreich durchaus vergleichbar - in den vergangenen 20 Jahren kaum geändert hat und bei etwa 0,3 Prozent der Bevölkerung liegt.

\section{ERST DIE DIAGNOSE, DANN DIE THERAPIE}

In der Medizin sollte man immer dem Grundsatz folgen: erst die Diagnose, dann die Therapie. In einem Abweichen davon liegt eine der Ursachen der US-Krise mit Opioiden, wo nachweislich auch Patient*innen mit Kopfschmerzen starke Opioide erhielten, obwohl sie bei Kopfschmerz-Diagnosen laut Evidenz unwirksam sind.
Die moderne Schmerzmedizin befolgt wissenschaftlich begründete Therapiegrundsätze. Tumorschmerzen, postoperative Schmerzen, Schmerzen nach Verletzungen und Entzündungsschmerzen sind gut belegte Indikationen mit zugrunde liegenden Diagnosen für den wirksamen Einsatz von Opioiden. Bei Nicht-Tumorschmerzen gab es eine umfangreiche fachlich-wissenschaftliche Debatte, die etwa in Deutschland zu einer Leitlinie (,,LONTS“) mit Diagnosen, Therapiechance und -dauer und in Österreich zu einem Positionspapier von Schmerz- und Suchtexpert*innen geführt hat. Von jahrelangem Einsatz starker Opioide bei vielen Personen mit NichtTumorschmerzen wird klar abgeraten.

In Europa, und speziell in Deutschland und Österreich, hat man früh auf Warnsignale reagiert. Eine Epidemie an Opioid-Fehlgebrauch ist daher ausgeblieben. Es werden nur Einzelfälle von Abhängigkeiten durch Verschreibung berichtet. Dies wird auch so bleiben, wenn alle weiterhin umsichtig vorgehen und nur passende und belegte Schmerztherapien einsetzen. Starke Opioide haben dabei ihren Stellenwert. Aber eben nur nach passenden Diagnosen und nach Abwägung von Nutzen und Nebenwirkungen, sodass der oder die jeweilige Betroffene tatsächlich einen nachweislichen Nutzen davon hat.

Redaktion 